\title{
Psicoterapia e Medicina Geral e Familiar - o potencial da terapia cognitivo comportamental
}

Sílvia Carvalho*

\section{RESUMO}

O aumento da prevalência das perturbações mentais, os seus custos e os efeitos da crise económica no agravamento deste problema reforçam a necessidade de intervenção psicológica. A Organização Mundial de Saúde (OMS) indica que cerca de metade de todo o sofrimento associado a situações de saúde-doença, no Ocidente, é devido a perturbação mental, sobretudo a depressão e a ansiedade. A investigação internacional demonstra a efetividade da intervenção psicológica. O benefício da intervenção psicológica verifica-se num conjunto de outras patologias, não necessariamente relacionadas com a perturbação mental, como doenças cardiovasculares, doenças oncológicas, artrite reumatóide, diabetes, dor crónica, entre outras. Os ganhos obtidos permitem uma redução do recurso aos serviços e de consumo de medicamentos, uma maior adesão à terapêutica e facilitação da mudança de comportamentos. A intervenção de natureza cognitiva-comportamental apresenta custos reduzidos e elevada taxa de recuperação. $O$ enfoque, dado atualmente à saúde mental da população e a multiplicidade de patologias que advêm direta ou indiretamente desta problemática, despertou na autora o interesse em conhecer um tipo de psicoterapia que a pudesse ajudar a lidar com estas perturbações. Numa consulta de medicina geral e familiar o médico pode fazer uso de algumas técnicas, introduzindo a terapia cognitiva e comportamental, mas um conjunto estruturado de consultas com ordem temporal para a realização de psicoterapia é algo fundamental e paralelo à consulta médica, complementando-a. Sendo assim, a autora considera que a aplicabilidade destas técnicas de psicoterapia valoriza o médico, facilitando a abordagem da pessoa como um todo.

Palavras-chave: Terapia Cognitiva Comportamental; Medicina Geral e Familiar.

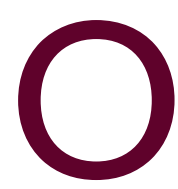
aumento da prevalência das perturbações mentais, os seus custos e os efeitos da crise económica no agravamento deste problema reforçam a necessidade de intervenção psicológica. A Organização Mundial de Saúde (OMS) indica que cerca de metade de todo o sofrimento associado a situações de saúde-doença, no Ocidente, é devido a perturbação mental, sobretudo a depressão e a ansiedade. ${ }^{1}$ A investigação internacional demonstra a efetividade da intervenção psicológica. Para além do alívio do sofrimento que a perturbação men-

* Médica interna de Medicina Geral e Familiar

Pós-graduação em Terapias Cognitivo Comportamentais no Instituto CRIAP - Psicologia e Formação Avançada

Unidade de Saúde Familiar Nascente - ACES Gondomar tal acarreta, este tipo de intervenções minimiza um conjunto de consequências sociais e económicas. O aumento da prevalência da perturbação mental na Europa e em Portugal são preocupantes. Em 2008, a União Europeia estimava que cerca de 50 milhões de pessoas (cerca de 11\% da população) tinham algum tipo de perturbação mental..$^{1-3}$ Em Portugal, relativamente à prevalência ao longo da vida, um em cada cinco cidadãos experienciou uma perturbação mental (23\%). ${ }^{1-3}$ Assim, Portugal apresenta uma prevalência de perturbações mentais acima da média europeia. Este problema assume particular relevância em contexto de crise económica. Um relatório recente da OMS sugere que os problemas a nível de saúde mental possam ser agravados pela crise económica, nomeadamente a existência 
de maior mortalidade, aumento da taxa de suicídio e do abuso crónico de álcool.

O benefício da intervenção psicológica verifica-se num conjunto de outras patologias, não necessariamente relacionadas com a perturbação mental, como doenças cardiovasculares, doenças oncológicas, artrite reumatóide, diabetes, dor crónica, entre outras. Os ganhos obtidos permitem uma redução do recurso aos serviços e de consumo de medicamentos, uma maior adesão à terapêutica e facilitação da mudança de comportamentos. A intervenção de natureza cognitiva-comportamental apresenta custos reduzidos e elevada taxa de recuperação..$^{4-6}$

Um estudo realizado em Portugal sobre a efetividade da psicoterapia permitiu concluir que $28 \%$ da amostra estudada recorreu a profissionais de ajuda, nomeadamente ao médico de família $(39,1 \%)$, seguindo-se o psiquiatra $(29,6 \%)$ e médicos de outras especialidades $(27,7 \%) .^{7}$ Os resultados encontrados colocam uma percentagem bastante considerável de pacientes a recorrer ao médico de família (MF) e permitem verificar que a maioria dos consumidores afirma ter melhorado e estar satisfeita com a intervenção psicológica desenvolvida, quando esta pressupõe terapia verbal. A intervenção baseada em psicofármacos está associada a piores índices de melhoria percebida e de satisfação. A melhoria do estado emocional geral é encontrada em $80 \%$ dos casos seguidos por psicólogos. Este valor é semelhante ao obtido por psiquiatras. A melhoria do estado emocional é menos referida quando o apoio é fornecido pelo MF (50,4\%). Este resultado é compreensível, tendo em conta o tempo que os MF dispõem para cada paciente, não sendo este por si só o único fator que justifica este descontentamento. Atualmente é necessário desenvolver mais estudos de custo-efetividade adaptados à realidade portuguesa, de modo a demonstrar a efetividade das intervenções psicológicas na redução dos gastos na saúde em Portugal.

O enfoque dado atualmente à saúde mental da população e a multiplicidade de patologias que advêm direta ou indiretamente desta problemática despertou na autora o interesse em conhecer um tipo de psicoterapia que a pudesse ajudar a lidar com estas perturbações.

A terapia cognitiva, também conhecida como terapia cognitiva comportamental (TCC), é um tipo espe- cífico de psicoterapia que enfatiza a importância dos processos cognitivos na compreensão e no tratamento de diversos transtornos mentais. Desenvolvida por Aaron Beck no final dos anos 1950, esta tornou-se uma das psicoterapias mais investigadas empiricamente e com mais evidências científicas de eficácia. ${ }^{8}$ As intervenções psicológicas breves nos centros de saúde permitem melhorar o funcionamento psicológico e reduzir em $50 \%$ o número de consultas médicas. ${ }^{9-10}$ A TCC é tão efetiva no tratamento da depressão e da ansiedade como a medicação, sendo preferida pela maioria dos doentes. ${ }^{11}$ Análises de custo-efetividade parecem apontar que a TCC seja tão efetiva, a curto prazo, como a terapêutica farmacológica para a depressão, ansiedade generalizada e outras perturbações mentais (fobias e stress pós-traumático). ${ }^{11}$ O NICE emitiu guidelines que estabelecem a TCC como opção no tratamento da depressão e ansiedade. Estas guidelines baseiam-se num conjunto de ensaios clínicos que indicam que a TCC é tão efetiva como tratamento farmacológico, a curto prazo, apresentando efeitos mais duráveis a longo prazo. ${ }^{12}$

O princípio básico da terapia cognitiva pode ser resumido da seguinte forma: as respostas emocionais e comportamentais, bem como a nossa motivação, não são influenciadas diretamente por situações, mas pela forma como processamos essas situações, por outras palavras, pelas interpretações que fazemos dessas situações ou pelo significado que lhes atribuímos. As interpretações, representações ou atribuições de significado, por sua vez, refletem-se em pensamentos automáticos. Estes pensamentos automáticos, pré-conscientes, refletem-se na ativação de estruturas básicas inconscientes: os esquemas e as crenças. Um exemplo simples para ilustrar este princípio é o seguinte: suponhamos que nos encontramos casualmente com um amigo que não nos cumprimenta. Se pensarmos "Ele não quer mais ser meu amigo", a emoção será tristeza e o comportamento será possivelmente afastarmo-nos do amigo. Se, porém, pensarmos "Oh, será que ele está aborrecido comigo?", a emoção será apreensão e o comportamento será procurar o amigo e perguntar o que se passa. Ou ainda, se pensarmos "Quem ele pensa que é para não me cumprimentar?", a emoção poderia ser raiva e o comportamento confrontar o amigo. Porém, diante da mesma situação, podemos ainda pensar "Não me cumprimentou... penso que não me viu"; e, nesse 
caso, as emoções e comportamentos seguiriam inalterados. Como podemos verificar, a mesma situação desencadeou várias interpretações e comportamentos.

Como em qualquer forma de psicoterapia, a TCC começa a avaliação através de uma anamnese completa e de um exame do estado mental do paciente. Na TCC, a avaliação e a realização da conceptualização é baseada num modelo amplo de tratamento. A conceptualização cognitiva, formulação de caso e enquadramento cognitivo funcionam como um mapa que orienta o trabalho a ser realizado com o paciente. ${ }^{15} \mathrm{O}$ terapeuta, através da conceptualização, visa obter a estrutura para a compreensão de cada paciente segundo a sua subjetividade, o que o auxilia na escolha de estratégias terapêuticas que são utilizadas ao longo do tratamento. ${ }^{14-15}$ Este tratamento exige várias sessões, sendo incompatível com a prática diária de um MF; no entanto, alguns conceitos da TCC podem aplicar-se à consulta e ao papel do MF. A TCC poderá ser uma formação complementar à prática clínica diária, mas exigirá ao médico um espaço e um tempo diferentes para a aplicação específica destas técnicas. No processo de terapia é importante que os doentes se consciencializem que esta é um complemento à consulta médica, com abordagens específicas, embora com o mesmo objetivo final. O médico que possui esta formação complementar tem uma mais-valia na sua aprendizagem como médico, pois enriquece e estreita a relação médico-doente, desenvolve uma maior acurácia diagnóstica, identificando as expectativas, significados, motivações e sentimentos expressos pelo utente, de forma a responder da melhor forma possível às necessidades destes mesmos utentes. A comunicação com o utente é uma das competências essenciais de qualquer médico, mas muito particularmente do MF. A especialidade de Medicina Geral e Familiar (MGF) é caracterizada, segundo a definição europeia, por seis competências nucleares, entre as quais se salienta o cuidado centrado na pessoa, uma abordagem do indivíduo com as suas crenças, medos, expectativas e necessidades. ${ }^{16} \mathrm{O}$ ato clínico por excelência é concretizável na relação terapêutica, da qual a entrevista é ferramenta essencial. As atividades preventivas são, em si, essencialmente uma tarefa comunicacional e que constituem uma parte fundamental da atividade do MF.

Relativamente à psicoterapia apreendida pela auto- ra, esta concedeu uma bagagem vasta de formas a abordar e ajudar os pacientes. Por vezes usamos as palavras "pensar" e "sentir" como sinónimos e na verdade não somos conscientes dos pensamentos automáticos que acompanham os sentimentos. Com um simples exercício na consulta de MGF os pacientes treinam, identificam e distinguem entre pensamentos e sentimentos. Podem também analisar-se as experiências perturbadoras e associadas a uma mudança no humor e na ligação destes com as suas respostas comportamentais. Por exemplo, se não age como gostaria de agir, poderão existir pensamentos ou crenças auto desmoralizantes, críticas ou perfecionismos que paralisam a ação: se estamos a exigir demasiada perfeição de um comportamento, isto pode criar tanta tensão que o mais certo é uma paralisação devido ao medo de falhar, como se esta "falha" fosse uma catástrofe. O objetivo final é desenvolver habilidades mentais para modificar processos cognitivos habituais mas, como acontece em qualquer habilidade, é preciso tempo e prática, sendo extremamente importante a adequação da intervenção a cada paciente. Numa consulta de MGF, o médico pode fazer uso de algumas técnicas, introduzindo a TCC, mas um conjunto estruturado de consultas com ordem temporal para a realização de psicoterapia é algo fundamental e paralelo à consulta médica. Sendo assim, a autora considera que a aplicabilidade destas técnicas de psicoterapia valoriza o médico, facilitando a abordagem da pessoa como um todo.

\section{AGRADECIMENTOS}

A autora agradece aos profissionais do Instituto CRIAP e o apoio da sua orientadora de formação e da Coordenação do Internato de Medicina Geral e Familiar da Zona Norte, sem os quais a realização deste estágio não teria sido possível.

\section{REFERÊNCIAS BIBLIOGRÁFICAS}

1. World Health Organization International Consortium in Psychiatric Epidemiology. Cross-national comparisons of the prevalence and correlates of mental disorders. Bull World Health Organ. 2000;78(4):41326.

2. World Health Organization. Impact of economic crisis on mental health. Copenhagen: Regional Office for Europe WHO; 2011.

3. Alto Comissariado da Saúde. Evolução dos indicadores PNS 20042010. Lisboa: Ministério da Saúde; 2010.

4. Layard R, Clark D, Knapp M, Mayraz G. Cost-benefit analysis of psychological therapy. London: Centre for Economic Performance, London School of Economics and Political Science; 2007. ISBN 9780853280941

5. Layard R. The depression report: a new deal for depression and anxie- 
ty disorders. London: Centre for Economic Performance, London School of Economics and Political Science; 2006.

6. Layard R. The case for psychological treatment centres. BMJ. 2006;332(7548):1030-2.

7. Branco-Vasco A, Santos O, Silva F. Psicoterapeuta sim! Eficácia, efetividade e psicoterapeutas (em Portugal). Psicologia. 2003;XVII(2):48595.

8. Alford BA, Beck AT. O poder integrador da terapia cognitiva. Porto Alegre: Artmed; 2000. ISBN 9788573077070

9. Romanow Commission. Costs and cost-offsets of psychological interventions: data in support of their integration into universal access health care system. Vancouver, BC: Romanow Commission; 2002.

10. Golden GA. Impact of psychotherapy: does it affect frequency of visits to family physicians? Can Fam Physician. 1997;43(1):1098-102.

11. Clark DM. Implementing the NICE guidelines for depression and anxiety: the IAPT experience. Int Rev Psychiatry. 2011;23(4):318-27.

12. National Institute for Clinical Excellence. National clinical practice guidelines: depression. London: NICE; 2005.

13. Beck AT, Rush AJ, Shaw BF, Emery G. Terapia cognitiva da depressão. Porto Alegre: Artmed; 1997. ISBN 9788573072488

14. Kuyken W, Fothergill CD, Musa M, Chadwick P. The reliability and qua- lity of cognitive case formulation. Behav Res Ther. 2005;43(9):1187201.

15. Persons JB, Roberts NA, Zalecki CA, Brechwald WA. Naturalistic outcome of case formulation-driven cognitive-behavior therapy for anxious depressed outpatients. Behav Res Ther. 2006;44(7):1041-51.

16. Allen J, Gay B, Crebolder H, Heyrman J, Svab I, Ram P, et al. A definição europeia de medicina geral e familiar: clínica geral/medicina familiar. EURACT; 2002.

\section{CONFLITO DE INTERESSES}

A autora declara não ter conflito de interesses.

\section{ENDEREÇO PARA CORRESPONDÊNCIA}

Sílvia Mariana Fonseca Carvalho

Unidade de Saúde Familiar Nascente

Rua Actor Mário Viegas S/N Piso 1

4435-076 Rio Tinto

E-mail: silviamarianacarvalho@gmail.com

Recebido em 06-08-2014

Aceite para publicação em 29-11-2014

\section{ABSTRACT}

\section{PSYCHOTHERAPY IN FAMILY MEDICINE: THE VALUE OF COGNITIVE BEHAVIORAL THERAPY}

The increasing prevalence of mental disorders, their costs, and the effects of the current economic crisis reinforce the need for psychological intervention in medical care. The World Health Organization (WHO) has stated that half of all the suffering associated with health and disease in Western countries is due to mental disorders, particularly depression and anxiety. Research has demonstrated the effectiveness of psychological interventions. The benefits of psychological intervention can occur in conditions not necessarily related to mental disorders such as cardiovascular diseases, cancer, rheumatoid arthritis, diabetes, chronic pain, and others. These benefits cause a reduction in the use of medical services and the use of medication, and promote greater adherence to therapy, as well as behavioral change. Cognitive therapy has low costs and a high success rate. Interest in mental health and the conditions caused by mental distress motivated the author to explore different types of psychotherapy that could be effective in primary care. The family physician can make use of some of the techniques of cognitive therapy. However time devoted to psychotherapy in parallel to the usual medical consultation may complement it. Application of CBT techniques in psychotherapy enhances medical practice and contributes to the whole-person approach.

Keywords: Cognitive Behavioral Therapy; Family Practice. 